\title{
Excitable dynamics in neural and cardiac systems
}

\author{
Roberto Barrio ${ }^{\mathrm{a}, *}$, Stephen Coombes ${ }^{\mathrm{b}}$, Mathieu Desroches ${ }^{\mathrm{c}}$, Flavio Fenton ${ }^{\mathrm{d}}$, \\ Stefan Luther ${ }^{\mathrm{e}, \mathrm{f}, \mathrm{g}}$, Esther Pueyo ${ }^{\mathrm{h}}$ \\ a IUMA and Applied Mathematics Department, University of Zaragoza, Zaragoza E-50009, Spain \\ ${ }^{\mathrm{b}}$ Centre for Mathematical Medicine and Biology, School of Mathematical Sciences, University of Nottingham, Nottingham NG7 2RD, UK \\ ${ }^{\mathrm{c}}$ MathNeuro Project-Team, Inria Sophia Antipolis Méditerranée, France \\ d School of Physics, Georgia Institute of Technology, Atlanta, GA,USA \\ e Max Planck Institute for Dynamics and Self-Organization, Göttingen 37077, Germany \\ ${ }^{\mathrm{f}}$ Institute of Pharmacology, University Medical Center, Georg-August-University, Göttingen, Germany \\ $\mathrm{g}$ German Center for Cardiovascular Research, Partner Site, Göttingen, Germany \\ ${ }^{\mathrm{h}}$ I3A, University of Zaragoza, IIS Aragón and CIBER-BBN, Zaragoza E-50018, Spain
}

\section{A R T I C L E I N F O}

\section{Article history:}

Available online $\mathrm{xxx}$

\section{MSC:}

92Bxx

\section{Keywords:}

Excitable dynamics

Neural systems

Cardiac systems

\begin{abstract}
A B S T R A C T
The application of mathematics, physics and engineering to medical research is continuously growing; interactions among these disciplines have become increasingly important and have contributed to an improved understanding of clinical and biological phenomena, with implications for disease prevention, diagnosis and treatment. This special issue presents examples of this synergy, with a particular focus on the investigation of cardiac and neural excitability.

This issue includes 24 original research papers and covers a broad range of topics related to the physiological and pathophysiological function of the brain and the heart. Studies span scales from isolated neurons and small networks of neurons to whole-organ dynamics for the brain and from cardiac subcellular domains and cardiomyocytes to onedimensional tissues for the heart.

This preface is part of the Special Issue on "Excitable Dynamics in Neural and Cardiac Systems".
\end{abstract}

(c) 2020 Elsevier B.V. All rights reserved.

\section{Introduction}

Neural tissue and heart muscle are important examples of biological excitable media. A key characteristic of an excitable medium is its capacity to allow the passage of electrical excitation from one element to another by means of local coupling. For neural and cardiac systems, this corresponds to their ability to respond strongly to the action of a relatively weak external stimulus delivered after a refractory period.

This special issue aims at providing an overview of recent theoretical and computational research in neural and cardiac systems. The basic elements of both systems are excitable cells, i.e., neurons and cardiac myocytes, which when connected form neural tissue and heart muscle, respectively, where new emergent behavior appears. The extensive use of mathematical and numerical techniques has allowed detailed studies to simulate the functioning of these two complex systems and to investigate the underlying mechanisms in both health and disease. As both systems share important characteristics but have

\footnotetext{
* Corresponding author.

E-mail addresses: rbarrio@unizar.es (R. Barrio), stephen.coombes@nottingham.ac.uk (S. Coombes), mathieu.desroches@inria.fr (M. Desroches), flavio.fenton@physics.gatech.edu (F. Fenton), stefan.luther@ds.mpg.de (S. Luther), epueyo@unizar.es (E. Pueyo).
} 
specific particularities, major objectives of this issue are, on the one hand, to show how both can be analyzed using similar techniques and, on the other hand, to showcase the dynamics of each particular system. The articles included use numerical modeling and time series analysis to elucidate the mechanisms underlying the onset, perpetuation, and control of complex neuronal and cardiac dynamics.

In summary, important contributions of this special issue include a snapshot of the current research landscape on these two types of excitable media and an emphasis on the numerous links between neural and cardiac studies using theoreti$\mathrm{cal} /$ mathematical and computational approaches.

\section{Neural systems}

The first group of papers is devoted to the study of neural systems across scales from detailed analysis of isolated neurons, small networks of neurons and finally large networks and brain studies.

\subsection{Isolated neuron dynamics}

Currently, stochastic models are increasingly common in mathematical neuroscience. In [1] the torus canard parametric region in the stochastic Hindmarsh-Rose neuron model is studied. It is shown that noise can transform the torus canard into a large-amplitude torus, which can underlie a transition from amplitude-modulated spiking behaviour to bursting.

The classical Hodgkin-Huxley (HH) and the FitzHugh-Nagumo (FHN) models are revisited in Rameh et al. [2], where the generation of the action potential is now achieved via a single delay-differential equation (DDE). The solutions of the new models are similar to those of the original $\mathrm{HH}$ and FHN models, showing that delay-differential equations can also be used as building blocks in the development of new mathematical cell models.

Fold/homoclinic (square-wave) bursting orbits are typical of many excitable dynamical systems. In [3] a complete theoretical scheme to describe spike-adding in these systems is given. The main bifurcations and their connection with homoclinic bifurcations are described. This global scheme provides an explanation of different phenomena presented in the literature (continuous and chaotic processes) and indicates where each kind of spike-adding process occurs in parameter space.

The firing properties of Purkinje cells are regulated by cerebellar stellate cells. These cells have a temporary increase in excitability during patch clamp configuration of the entire cell in what is called "runup". In [4] modeling approaches are used to clarify the dynamics of runup and classify the firing behavior of cerebellar stellate cells as type I oscillators that have a saddle node in an invariant cycle (SNIC) bifurcation.

Phase response curves (PRC) have been widely used to understand the response of limit cycle oscillators to weak external perturbation. Recently, extensions of the phase-oscillator reduction technique have been introduced to capture more information away from the limit cycle, including the phase response function (PRF, a generalization of the PRC) and the amplitude response function (ARF). In [5] the study of the dynamics of PRF-ARF systems under periodic pulsatile stimuli is presented using several techniques from dynamical systems.

Inner hair cells (IHCs) are excitable sensory cells in the inner ear that encode acoustic information. In [6] a threedimensional model of a reduced IHC is proposed and non-dimensionalization is carried out. When the typical time scales of the variables in the reduced IHC model are estimated, it is shown that this model can be characterized by two fast and one slow or one fast and two slow variables, depending on the biophysically relevant parameters that control the dynamics. This fast-slow analysis describes how to pass from pseudo-plateau bursting to mixed-mode oscillations by varying a single parameter in the model.

The phenomenon of reverse stochastic resonance (ISR) consists of an unexpected depression in the response of a system subject to external noise, for example, as seen in the average firing rate in some pacemaker neurons subject to moderate noise values. In [7], the specific conditions for ISR in a general bistable model system are determined. In addition, the existence of another non-standard stochastic resonance is shown, even in the absence of any weak input signal.

\subsection{Coupled neuron dynamics}

The study of the synchronization patterns of small neuron networks that control different biological processes has become a growing discipline. In [8-10] the problem of studying small groups of neurons, and more specifically patterns in central pattern generators (CPGs), is treated.

In [8] the problem of the detection of patterns in generic CPGs is studied and two new numerical techniques are introduced: a lateral phase delay analysis based on a graphical representation of some Poincaré maps and a sweep quasi-Monte Carlo method with an optimized generic classification of the different patterns that allows location and classification of all coexisting rhythms. As a test example, the authors consider an insect movement CPG consisting of six coupled neurons for cockroach motor neurons.

In [9] the coexistence of multistable rhythms generated by oscillatory neural circuits formed by four cells is studied. Modern computational approaches are used that include unsupervised machine-learning algorithms and fast parallel 
simulations on GPUs, which allow the authors to analyze the mechanisms that establish the robustness and multifunctionality of these neural circuits.

In [10], using a phase-reduction technique of an intersegmental network model of stick insect locomotion, the authors construct a reduced model of the intra- and intersegmental network that controls the activity of the depressor elevator in the meso- and metathoracic ganglia. When examining intra- and intersegmental phase differences in the model, the authors identified the properties of network couplings that replicate several results observed in stick insect experiments. In addition, they define general characteristics of coupling between CPGs of any type that maintain the phase relationships observed in experiments.

Analyzing spike sequences generated by individual and coupled stochastic FitzHugh-Nagumo models shows that relative spike timing can encode signal information [11]. Using a symbolic method to analyze the sequence of spikes, preferred and uncommon spike patterns can be detected. It is found that for a group of neurons, only a few connections to each other can significantly improve the coding of small-amplitude signals, which indicates that the encoding of information in preferred and that unusual spike patterns are a plausible way for neuronal populations to encode weak periodic inputs, exploiting the presence of neuronal noise.

The theory of communication through coherence (CTC) proposes that brain oscillations reflect changes in the excitability of neurons and, therefore, successful communication between two oscillating neural populations. A population rate model consisting of excitatory and inhibitory cells is considered in [12]. Different bifurcation studies are performed as several phase-locked states are detected, as well as bistability regions. The neuronal implications of these findings are studied in detail.

Stochastic resonance (SR) is a noise-induced phenomenon reported in dynamical systems with a threshold, while reverse stochastic resonance (ISR) is the opposite phenomenon that occurs in dynamical systems with bistability between a stable node and a stable limit cycle. In [13] the possible coexistence of both phenomena, SR and ISR, is shown in a minimal circuit of neurons that are synaptically coupled. Simulations indicate that the main characteristics of SR are the direct results of dynamic properties that lead to ISR.

\subsection{Brain dynamics}

The study of brain electrical activity measured by electroencephalographic recordings (EEG) is an important technique for the understanding and diagnosis of neurological diseases. Several applications to detect pathologies or to study brain waves are shown in David et al. [14], Galadí et al. [15]. In [14], multifractal deviated fluctuation analysis, the Hurst exponent (H) and the fractal dimension (D) are used to analyze the dynamics of EEG signals from normal and epileptic patients using a set of physiological time series. In addition, a new measure is proposed, the combined index. The results presented indicate that the $\mathrm{H}$ and $\mathrm{D}$ indices are useful for constructing a combined measure to study healthy and epileptic brain EEG activity. In [15] the authors focus on understanding EEG data from first principles through numerical simulation. They study a network model of excitatory and inhibitory neurons that generates a variety of comparable waves similar to $\alpha, \beta, \gamma$ and other rhythms observed in EEG recordings.

The brain can be considered a system that optimizes the structure of anatomical connections based on efficiency requirements of functional connectivity. The power of this principle in organizing the complexity of brain architecture is illustrated in Tapia et al. [16], where functional connectivity is described as diffusion in the current network structure. By showing how several structures arise (modular small-worlds with rich club effects, as observed in neural anatomy), the authors offer a new conjecture about how structures similar to natural and artificial brains can be physically implemented.

The responses of excitable systems can be probed by exposing them to a variety of different perturbations. Typically, small inputs produce approximately linear responses, whilst large ones may give rise to radically different large amplitude nonlinear responses. A difficult task is to determine the transition between the two. In [17] this transition, between normal and pathological responses, is studied using a variety of mathematical techniques in a model of coupled Wendling neural masses.

The gamma rhythm $(20-100 \mathrm{~Hz})$ is present in numerous cognitive tasks. A weak gamma rhythm results from synchronous firing of pyramidal neurons in an interaction with local inhibitory interneurons in a pyramidal-interneuron gamma (PING). Based on previous results, the authors of [18] postulate that dopaminergic modulation can control weak gamma clustering and explain how dopaminergic network modulation and cellular properties influence the cluster formation process in a PING network model.

Mammals can efficiently select actions based on noisy signals and adapt to unexpected results to better take advantage of future opportunities. These behavioral changes based on feedback depend partially on long-term plasticity within corticobasal-ganglia-thalamic networks, driven by dopaminergic modulation. In [19], the authors simulate the evolution of synaptic corticostriatal weights based on a spike-time-dependent plasticity rule driven by dopamine signaling that is induced by the results of actions in the context of a task of forced choice between two alternatives.

The estimation of parameters in stochastic processes has been investigated for decades and the asymptotic properties of the estimators are known. However, achieving the regime where asymptotic properties are valid can take so long that well-founded theoretical results have no practical value. In [20], three processes of the Pearson diffusion class are studied, which are examples of leaky integrate-and-fire models, that describe the electrical properties of a neuronal membrane. 


\section{Cardiac systems}

The second group of papers is devoted to the study of cardiomyocytes exhibiting arrythmic dynamics and the propagation of waves in one-dimensional cardiac tissue fibers and generic excitable media.

The study presented in Lai et al. [21] sheds light on the phenomenon of cardiac alternans, which has been reported to increase the risk for cardiac arrhythmias in different clinical scenarios. Cardiac cellular alternans are defined as a period2 oscillatory response of the membrane potential or the intracellular calcium concentration. Along with calcium alternans developing uniformly in the whole cardiomyocyte, subcellular calcium alternans has been also described, which manifests as spatial heterogeneities in the amplitude of the calcium transients along a cardiomyocyte. A two-dimensional network of calcium release units in cardiomyocytes is used to investigate the role of calcium buffers and L-type calcium channels in the generation of subcellular calcium alternans. By numerical simulations, calcium diffusion in the sarcoplasmic reticulum is shown to be a critical determinant of the spatial pattern of subcellular calcium alternans.

In [22], complex cardiac excitation dynamics at macroscopic scales is investigated by methods accounting for non-local spatio-temporal coupling, which presents the advantage of avoiding the need for resolution of tissue heterogeneities to microscopic scales. Specifically, a simulation study of fractional diffusion models in one-dimensional cardiac tissue fibers is presented, with both phenomenological and biophysically detailed models used to represent the electrophysiology of ion channels. The authors prove that spatio-temporal features of cardiac tissue are influenced by the introduction of non-local operators in such a way that the domain size plays a much more important role in the model output than in the standard local case. Importantly, the effects of boundary conditions are not only relevantly observed near the boundaries of the domain but in a much wider space due to the introduced non-locality.

The work presented in Bezekci and Biktashev [23] offers a characterization of the relationship between the strength and duration of stimulation in one-dimensional excitable media. Threshold phenomena are important because they determine whether cardiac action potentials in the heart can be elicited or not and, consequently, whether success or failure in electrical propagation occurs. Similarly, threshold phenomena have been reported to be key in explaining pathological findings in brain diseases such as Alzheimer. Here, the authors focus on strength-duration curves and propose an analytical approach to assess ignition of propagation waves in one-dimensional media like those from the brain or the heart, including both linear and quadratic approximations. Subsequently, a "hybrid" approach is proposed where the ingredients of the expressions used in those approximations are numerically computed. Applicability of the proposed methods is illustrated for five different models, covering one-component as well as multi-component examples.

In [24], novel adaptive time-step numerical methods are proposed to solve stiff excitable models that use Markov chain formulations to describe ion channel dynamics. This type of models is becoming increasingly used to simulate electrical abnormalities due to mutations in genes encoding ion channels or to predict the effects of drug-channel interactions. Since many of these models are stiff, the performance of standard explicit solvers, like the Euler method, is compromised due to stability constraints. Among the methods proposed in the study, the adaptive time-step scheme that combines the generalized Rush-Larsen method with Uniformization (adaptive GLR1 + UNI) is shown to outperform all other tested methods in a range of scenarios involving highly stiff cellular models. In single cell simulations, the adaptive GLR1 + UNI is more than 25 times faster than the adaptive time-step Euler method and 350 times faster than the fixed time-step Rush-Larsen method. In 3D tissue simulations, it renders a 5-fold acceleration with respect to the adaptive Euler method.

\section{Acknowledgments}

RB has been supported by the Spanish Ministry of Economy and Competitiveness (grant PGC2018-096026-B-I00), European Social Fund (EU) and Aragón Government (grant LMP124-18 and group E24-17R), and the University of Zaragoza-CUD (grant UZCUD2019-CIE-04).

SC has been supported by the Engineering and Physical Sciences Research Council (grant EP/P007031/1).

FHF has been supported by NSF (grants CNS-1446675 and CMMI-1762553) and NIH (grant 1R01HL143450-01).

S.L. acknowledges support by the German Center for Cardiovascular Research (DZHK), by the German Research Foundation through SFB 1002 Modulatory Units in Heart Failure, and by the Max Planck Society.

EP has been supported by the European Research Council (grant ERC-638284), Spanish Ministry of Economy and Competitiveness (grant DPI2016-75458-R), European Social Fund (EU) and Aragón Government (grant LMP124-18 and group T39-17).

\section{References}

[1] Ryashko L, Slepukhina E. Noise-induced toroidal excitability in neuron model. Commun Nonlinear Sci Numer Simul 2020;82:105071. doi:10.1016/j. cnsns.2019.105071.

[2] Rameh RB, Cherry EM, dos Santos RW. Single-variable delay-differential equation approximations of the Fitzhugh-Nagumo and Hodgkin-Huxley models. Commun Nonlinear Sci Numer Simul 2020;82:105066. doi:10.1016/j.cnsns.2019.105066.

[3] Barrio R, Ibáñez S, Pérez L, Serrano S. Spike-adding structure in fold/hom bursters. Commun Nonlinear Sci Numer Simul 2020;83:105100. doi:10.1016/ j.cnsns.2019.105100.

[4] Mitry J, Alexander RP, Farjami S, Bowie D, Khadra A. Modeling excitability in cerebellar stellate cells: temporal changes in threshold, latency and frequency of firing. Commun Nonlinear Sci Numer Simul 2020;82:105014. doi:10.1016/j.cnsns.2019.105014.

[5] Castejón O, Guillamon A. Phase-amplitude dynamics in terms of extended response functions: invariant curves and Arnold tongues. Commun Nonlinear Sci Numer Simul 2020;81:105008. doi:10.1016/j.cnsns.2019.105008. 
[6] Baldemir H, Avitabile D, Tsaneva-Atanasova K. Pseudo-plateau bursting and mixed-mode oscillations in a model of developing inner hair cells. Commun Nonlinear Sci Numer Simul 2020;80:104979. doi:10.1016/j.cnsns.2019.104979.

[7] Torres JJ, Uzuntarla M, Marro J. A theoretical description of inverse stochastic resonance in nature. Commun Nonlinear Sci Numer Simul 2020;80:104975. doi:10.1016/j.cnsns.2019.104975.

[8] Barrio R, Lozano A, Rodríguez M, Serrano S. Numerical detection of patterns in CPGs: gait patterns in insect movement. Commun Nonlinear Sci Numer Simul 2020;82:105047. doi:10.1016/j.cnsns.2019.105047.

[9] Pusuluri K, Basodi S, Shilnikov A. Computational exposition of multistable rhythms in 4-cell neural circuits. Commun Nonlinear Sci Numer Simul 2020;83:105139. doi:10.1016/j.cnsns.2019.105139.

[10] Yeldesbay A, Daun S. Intra- and intersegmental neural network architectures determining rhythmic motor activity in insect locomotion. Commun Nonlinear Sci Numer Simul 2020;82:105078. doi:10.1016/j.cnsns.2019.105078.

[11] Masoliver M, Masoller C. Neuronal coupling benefits the encoding of weak periodic signals in symbolic spike patterns. Commun Nonlinear Sci Numer Simul 2020;82:105023. doi:10.1016/j.cnsns.2019.105023.

[12] Pérez-Cervera A, Seara TM, Huguet G. Phase-locked states in oscillating neural networks and their role in neural communication. Commun Nonlinear Sci Numer Simul 2020;80:104992. doi:10.1016/j.cnsns.2019.104992.

[13] Zamani A, Novikov N, Gutkin B. Concomitance of inverse stochastic resonance and stochastic resonance in a minimal bistable spiking neural circuit. Commun Nonlinear Sci Numer Simul 2020;82:105024. doi:10.1016/j.cnsns.2019.105024.

[14] David S, Machado J, Inácio C, Valentim C. A combined measure to differentiate EEG signals using fractal dimension and MFDFA-Hurst. Commun Nonlinear Sci Numer Simul 2020;84:105170. doi:10.1016/j.cnsns.2020.105170.

[15] Galadí JA, Torres JJ, Marro J. Emergence and interpretation of oscillatory behaviour similar to brain waves and rhythms. Commun Nonlinear Sci Numer Simul 2020;83:105093. doi:10.1016/j.cnsns.2019.105093.

[16] Tapia CC, Makarov VA, van Leeuwen C. Basic principles drive self-organization of brain-like connectivity structure. Commun Nonlinear Sci Numer Simul 2020;82:105065. doi:10.1016/j.cnsns.2019.105065.

[17] Hebbink J, van Gils SA, Meijer HG. On analysis of inputs triggering large nonlinear neural responses slow-fast dynamics in the Wendling neural mass model. Commun Nonlinear Sci Numer Simul 2020;83:105103. doi:10.1016/j.cnsns.2019.105103.

[18] Zakharov D, Krupa M, Gutkin B. Modeling dopaminergic modulation of clustered gamma rhythms. Commun Nonlinear Sci Numer Simul 2020;82:105086. doi:10.1016/j.cnsns.2019.105086.

[19] Vich C, Dunovan K, Verstynen T, Rubin J. Corticostriatal synaptic weight evolution in a two-alternative forced choice task: a computational study. Commun Nonlinear Sci Numer Simul 2020;82:105048. doi:10.1016/j.cnsns.2019.105048.

[20] Ditlevsen S, Rubio AC, Lansky P. Transient dynamics of Pearson diffusions facilitates estimation of rate parameters. Commun Nonlinear Sci Numer Simul 2020;82:105034. doi:10.1016/j.cnsns.2019.105034.

[21] Lai YM, Coombes S, Thul R. Calcium buffers and L-type calcium channels as modulators of cardiac subcellular alternans. Commun Nonlinear Sci Numer Simul 2020;85:105181. doi:10.1016/j.cnsns.2020.105181.

[22] Cusimano N, Gizzi A, Fenton F, Filippi S, Gerardo-Giorda L. Key aspects for effective mathematical modelling of fractional-diffusion in cardiac electrophysiology: a quantitative study. Commun Nonlinear Sci Numer Simul 2020;84:105152. doi:10.1016/j.cnsns.2019.105152.

[23] Bezekci B, Biktashev V. Strength-duration relationship in an excitable medium. Commun Nonlinear Sci Numer Simul 2020;80:104954. doi:10.1016/j. cnsns.2019.104954

[24] Gomes JM, Oliveira RS, Lobosco M, dos Santos RW. Adaptive-step methods for Markov-based membrane models. Commun Nonlinear Sci Numer Simul 2020;85:105249. doi:10.1016/j.cnsns.2020.105249. 